\title{
The Association between the LPA Gene Polymorphism and Coronary Artery Disease in Chinese Han Population
}

\author{
Zi-Kai Song, Hai-Di Wu, Hong-Yan Cao, and Ling Qin \\ Department of Cardiology, The First Hospital of Jilin University, 3302 JiLin Street, Changchun 130031, China \\ Correspondence should be addressed to Ling Qin; qinling1958@aliyun.com
}

Received 23 January 2014; Accepted 26 February 2014; Published 25 March 2014

Academic Editor: Cristiano Capurso

Copyright ( $2014 \mathrm{Zi}$-Kai Song et al. This is an open access article distributed under the Creative Commons Attribution License, which permits unrestricted use, distribution, and reproduction in any medium, provided the original work is properly cited.

\begin{abstract}
Lp(a) has been well known as an independent risk factor for coronary artery disease (CAD). The LPA gene, as it encodes apo(a) of the $\mathrm{Lp}$ (a) lipoprotein particle, was associated with increased risk of CAD. The purpose of this study was to analyze the relationship between the polymorphisms of LPA gene and CAD in Chinese Han population. Five SNPs (rs1367211, rs3127596, rs6415085, rs9347438, and rs9364559) in the LPA gene were genotyped using Sequenom MassARRAY time-of-flight mass spectrometer (TOF) in $560 \mathrm{CAD}$ patients as case group and 531 non-CAD subjects as control group. The numbers of these two groups were from Chinese Han ancestry. The results showed that allele $(P=0.046)$ and genotype $(P=0.026)$ of rs 9364559 in the $L P A$ gene was associated with CAD. The frequency of rs9364559 minor allele $(G)$ in case group was obviously higher than that in control group. Results of haplotype analysis showed that 4 haplotypes which contained rs9364559-G were associated with increased risk of CAD in this population. This study explored rs9364559 in the LPA gene may be associated with the pathogenesis of CAD; and the risk of CAD might be higher in the population carrying 4 haplotypes of different blocks in the LPA gene.
\end{abstract}

\section{Introduction}

Coronary artery disease (CAD) has become a major cause of death and disability, accounting for up to $40 \%$ of all lethal events [1]. However, the pathogenesis of CAD is not fully understood yet. Many studies showed that CAD is mainly caused by genetic and environmental factors [2]. Many genomewide association studies (GWAS) have identified several novel susceptibility gene loci for CAD [3-6]. Variants of lipid metabolism-related genes have received the widespread attention of scientists.

Elevated $\mathrm{Lp}(\mathrm{a})$ levels has been considered to be an independent risk factor for CAD [7-10]. In 1963, Berg first discovered the lipoprotein (a) $(\operatorname{Lp}(a))$ in the separation of plasma lipoprotein [11]. $\mathrm{Lp}(\mathrm{a})$ is produced in the liver [12] and circulates in the plasma, which is an LDL-like particle that consists of an apolipoprotein (a) (apo(a)) moiety linked to one molecule of apolipoprotein B100 via a disulfide bond [13]. The study by Boerwinkle et al. shown that the plasma $\mathrm{Lp}(\mathrm{a})$ concentration was mainly affected by apo(a) gene polymorphism, which accounts for $91 \%$ of the variation [14].
A genome wide association study showed that a cluster of genes-solute carrier family 22 member 3 (SLC22A3), lipoprotein (a)-like 2 (LPAL2), and lipoprotein (a) (LPA) on chromosome 6q26-27 was strongly associated with CAD risk [15]. $L P A$ gene in the 6q26-27 region encodes apo(a) of the Lp(a) lipoprotein particle [9]. Several LPA gene polymorphisms have been identified that have significant associations with an elevated $\operatorname{Lp}(\mathrm{a})$ level and a reduced copy number of $\mathrm{K} 4$ repeats [16]. However, the relationship between $L P A$ gene polymorphisms and CAD varies across races/ethnicities as there are large differences between minor allele frequencies. In the study by Clarke et al. rs3798220 and rs10455872 are strongly associated with serum Lp(a) levels in Caucasians [9]. However, the variant allele frequency (G allele) of rs10455872 is less than $1 \%$ in Chinese people without CAD. Another LPA SNP rs6415084, within the same haplotype block as the KIV-2 variation, was significantly associated with both Lp(a) concentration and KIV-2 copy number in the same direction in all 3 ethnicities. Therefore, the objective of our study was to investigate a possible association between $L P A$ gene polymorphisms and the risk of CAD in a case-control study of the Chinese Han population. 


\section{Materials and Methods}

2.1. Study Subjects. All subjects included 560 CAD patients (308 males and 252 females) and 531 controls (270 males and 261 females) in order to undertake a genetic analysis for association between the LPA gene polymorphisms and CAD. All the subjects including the case group and the control group used for this study were Chinese of Han descent. The case group was collected from the hospitalized patients with CAD in the cardiology department of the First Hospital of Jilin University from June 2009 to September 2012.

Diagnose was carried independently by at least two welltrained physicians based on the following criteria. All patients were identified with CAD by coronary computed tomographic angiography (SIEMNS Somatom Definition AS+128 row spiral CT). CAD was defined by $\geq 50 \%$ stenosis in any major coronary artery. All recruited patients had evidence of CAD documented by unstable angina or myocardial infarction. Unstable angina and myocardial infarction were confirmed by Chinese guidelines (Chinese Medical Cardiology Subcommittee, Chinese Editorial Committee of Cardiology Journal, 2010; Chinese Medical Cardiology Subcommittee, Chinese Editorial Committee of Cardiology Journal, 2007). Patients with nonatherosclerotic vascular diseases, congenital heart disease, cardiomyopathy, valvular disease, renal or hepatic disease, and cancer were excluded. All control subjects had ECG, chest X-ray, and serum analysis. They were classified as healthy subjects based on their normal physical examination results coupled with the absence of personal or family history and reasons for being suspected CAD.

The presence of cardiovascular risk factors, including diabetes mellitus (fasting blood glucose $\geq 7.0 \mathrm{mmol} / \mathrm{L}$ and/or using glucose-lowering medication, including insulin), blood pressure, and cigarette smoking, were obtained from all participants. Hypertension was defined according to seated blood pressure readings of $140 / 90 \mathrm{mmHg}$ and higher and/or subjects' receiving antihypertensive medication. In this study, hypercholesterolemia was defined as a serum total cholesterol level of $200 \mathrm{mg} / \mathrm{dL}$ or more, and a smoking habit was defined as a daily intake of $>10$ cigarettes [17].

All the subjects have written informed consent for the study, which was approved by ethics committee of Jilin University, Changchun, China.

2.2. Laboratory Examination. Before starting the study, all participants underwent an initial screening assessment that included medical history, vital signs, a 12-lead electrocardiogram, and measurement of lipid variables and novel risk factors. Venous blood was collected in the morning after an overnight (8-12 hours) fast. Serum/plasma samples were frozen and stored at $-80^{\circ} \mathrm{C}$ prior to analysis. All measurements were performed in a central laboratory.

2.3. SNP Selection, Identification, and Genotyping. Tagging SNPs were chosen from genotyped SNPs in Chinese Han population (CHB) of the HapMap project (Phase I database). The candidate SNPs were restricted to minor allele frequency bigger than 15\% in HAPMAP-CHB database (http://www.hapmap.org/). Genomic DNA used for PCR amplification was extracted from the whole blood sample using a DNA extraction kit (Takara, China). Primers of amplification and extension were used AssayDesigner3.1 software. Amplification and extension primers sequences of five loci in $L P A$ gene were in Table 1. Genotypes were assigned real time using Typer 4.0 software (Sequenom). As quality controls, $5 \%-10 \%$ of the samples were genotyped in duplicate. No inconsistencies were observed. Controls distributed in each 384 well plates were also consistent. Cluster plots were made of the signals from the low and the high mass allele.

2.4. Statistical Analysis. Data were expressed as percentages of total for categorical variables or mean \pm SD. The statistical analyses on the characteristics of the subjects were performed with Pearson $\chi^{2}$ test for the categorical variables such as sex, smokers, drinkers, hypertension, and diabetes and with Student's $t$-test for the continuous variable of age, TC, and TG with normal distribution. SPSS 16.0 was used for the above analyses.

The Hardy-Weinberg equilibrium for the genotypic distributions of SNPs was tested by the chi-square $\left(\chi^{2}\right)$ goodnessof-fit test. The Haploview program (version 4.1) was applied to estimate the linkage disequilibrium (LD) measures $\left(D^{\prime}\right.$ and $r^{2}$ ) between paired SNPs. Allelic, genotypic, and haplotype analyses were performed with SHEsis software. Software website is http://analysis.bio-x.cn/. Results are expressed as odds ratio (OR) and $95 \%$ confidence intervals (CI). $P<0.05$ was used as the criterion of statistical significance, and all statistical tests were two sided.

\section{Results}

3.1. Characteristics of Participants. In Table 2, the demographic and clinical characteristics of the 560 CAD patients and 531 control subjects have been listed. There was no significant difference of mean ages, sex, BMI, and serum TG level between case and control groups. However, compared with control group, CAD group had more smokers and more individuals with hypertension and diabetes. Additionally, compared with control group, CAD group had higher level of serum TC.

3.2. Allele and Genotype Analysis. The distributions of alleles and genotypes of five loci among participants were presented in Tables 3 and 4, respectively. Analysis with the SHEsis software showed that genotype and allele frequencies of rs9364559 were significantly higher in case group than that in control group, respectively $\left(\chi^{2}=7.302, P=0.026 ; \chi^{2}=\right.$ $3.981, P=0.046)$. However, for another four SNPs, there were no differences in genotype and allele frequencies between two groups.

3.3. Linkage Disequilibrium (LD) Analysis. Rs1367211 and rs6415085, rs1367211 and rs9347438, and rs3127596 and rs9364559 are all located in different LD block on 6q26-27 region $\left(D^{\prime}=1.000, r^{2}=0.085 ; D^{\prime}=1.000, r^{2}=0.149 ; D^{\prime}=\right.$ $\left.0.999, r^{2}=0.082\right)$. 


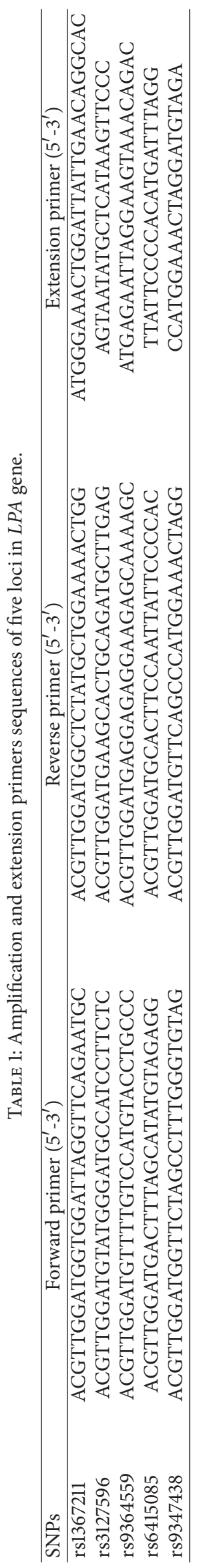


TABLE 2: Base characteristics of case group and control group.

\begin{tabular}{lccr}
\hline Variable & Case group $(n=560)$ & Control group $(n=531)$ & $P$ value \\
\hline Age (year) & $62.39 \pm 10.90$ & $61.82 \pm 12.77$ & 0.447 \\
Sex (\%) & 55.0 & 50.8 & 0.170 \\
Smoking (\%) & 38.3 & 23.3 & 0.000 \\
Drinking (\%) & 22.3 & 22.7 & 0.903 \\
Hypertension (\%) & 56.1 & 30.7 & 0.000 \\
Diabetes mellitus (\%) & 24.0 & 12.1 & 0.000 \\
TC $(\mathrm{mmol} / \mathrm{L})$ & $5.22 \pm 4.28$ & $4.62 \pm 1.24$ & 0.002 \\
TG $(\mathrm{mmol} / \mathrm{L})$ & $1.76 \pm 1.22$ & $1.74 \pm 1.20$ & 0.962 \\
BMI $\left(\mathrm{kg} / \mathrm{m}^{2}\right)$ & $24.19 \pm 2.68$ & $24.01 \pm 3.31$ & 0.431 \\
\hline
\end{tabular}

TABLE 3: SNPs loci allelic frequency distribution of LPA gene and the relationship with coronary heart disease.

\begin{tabular}{lcccccc}
\hline \multirow{2}{*}{ SNPs } & \multicolumn{2}{c}{ Controls } & \multicolumn{2}{c}{ Cases } & \multirow{2}{*}{$\chi^{2}$} & $P$ \\
& A & G & A & G & & \\
\hline rs1367211 & 204 & 842 & 216 & 860 & 0.109 & 0.741 \\
rs3127596 & 889 & 157 & 914 & 170 & 0.186 & 0.667 \\
rs9364559 & 738 & 306 & 716 & 358 & 3.981 & 0.046 \\
\hline & G & T & G & T & & \\
\hline rs6415085 & 769 & 257 & 796 & 280 & 0.262 & 0.609 \\
\hline & $\mathrm{C}$ & $\mathrm{T}$ & $\mathrm{C}$ & $\mathrm{T}$ & & \\
\hline rs9347438 & 395 & 653 & 407 & 671 & 0.001 & 0.976 \\
\hline
\end{tabular}

TABLE 4: SNPs loci genotype frequency distribution of $L P A$ gene and the relationship with coronary heart disease.

\begin{tabular}{lcccccccc}
\hline \multirow{2}{*}{ SNPs } & \multicolumn{3}{c}{ Controls } & \multicolumn{3}{c}{ Cases } & \multirow{2}{*}{$\chi^{2}$} & $P$ \\
& AA & GA & GG & AA & GA & GG & & \\
\hline rs1367211 & 23 & 158 & 342 & 20 & 176 & 342 & 0.967 & 0.616 \\
rs3127596 & 379 & 131 & 13 & 387 & 140 & 15 & 0.186 & 0.911 \\
rs9364559 & 266 & 206 & 50 & 231 & 254 & 52 & 7.302 & 0.026 \\
\hline & GG & GT & TT & GG & GT & TT & & \\
\hline rs6415085 & 346 & 77 & 90 & 352 & 92 & 94 & 1.574 & 0.455 \\
\hline & CC & TC & TT & CC & TC & TT & & \\
\hline rs9347438 & 75 & 245 & 204 & 77 & 253 & 209 & 0.004 & 0.998 \\
\hline
\end{tabular}

3.4. Haplotype Association Analysis. All haplotypes with a frequency above 3\% were included in the following analysis. In the haplotype association analysis, one haplotype was treated as a single variant, and all the other haplotypes were collapsed into the alternative allele to test its association with CAD. Four haplotypes formed by different blocks were associated with increased risk of CAD in this population (Table 5). GATG (which was made up by rs1367211, rs3127596, rs6415085, and rs9364559), ATG (which was made up by rs3127596, rs9347438, and rs9364559), GTG (which was made up by rs1367211, rs9347438, and rs9364559), and TG (which was made up by rs6415085 and rs9364559) reached the singlepoint significance level $(P=0.034,0.036,0.049$, and 0.039$)$.

\section{Discussion}

Based on our results, we found the association between rs9364559 in the LPA gene and risk of CAD in Chinese Han population; haplotypes GATG (which was made up by rs1367211, rs3127596, rs6415085, and rs9364559), ATG (which was made up by rs3127596, rs9347438, and rs9364559), GTG (which was made up by rs1367211, rs9347438, and rs9364559), and TG (which was made up by rs6415085 and rs9364559) were risk haplotypes for CAD in Chinese Han population. Therefore, rs9364559 in the LPA gene has played a significant role in the pathogenesis of CAD in Chinese Han population. This result agrees with the previous results in other populations $[9,18]$. However, the result that rs9364559 was associated with CAD in Chinese Han population is reported for the first time.

High level of $\operatorname{Lp}(\mathrm{a})$ in plasma has been confirmed to be associated with an increased cardiovascular risk [9, 1923], which is predominantly determined by apo(a) gene [14]. Apo(a) size polymorphism and nonsize polymorphism in the LPA gene all affect Lp(a) level [24, 25]. Rs3798220, located in the protease-like domain of apo(a), and rs10455872, which maps to intron 25, have repeatedly been associated with an increased Lp(a) level and a reduced copy number of K4 repeats in Caucasians [9]. These variants were both associated with plasma Lp(a) levels, and the association between these gene variants and CHD was abolished when plasma $\mathrm{Lp}(\mathrm{a})$ levels were entered into the model [9]. However, the study by Lamon-Fava et al. showed that rs3798220 was significantly associated with $\mathrm{Lp}(\mathrm{a})$ levels, and it was not a significant predictor of CHD [26]. And the association between apo(a) gene polymorphism and $\mathrm{CAD}$ varies among races. Both of these SNPs had no significant effect on serum Lp(a) levels in Chinese Han population [27]. Our study found that genotype and allele frequencies of rs9364559 in the LPA gene were significantly higher in case group than that in control group, respectively. Therefore, rs9364559 might affect risk of CAD. In this study, 4 haplotypes formed by different blocks in the LPA gene were risk haplotypes for CAD in Chinese Han population, and these haplotypes all contain rs9364559-G. Consequently, the above results further proved that LPA gene is associated with $\mathrm{CAD}$, which is consistent with previous studies. 
TABLE 5: Haplotype analysis of all blocks between two groups.

\begin{tabular}{|c|c|c|c|c|c|c|}
\hline Haplotype & case & control & $\chi^{2}$ & $P$ & OR & $95 \% \mathrm{CI}$ \\
\hline \multicolumn{7}{|c|}{ rs1367211, rs3127596, rs6415085, rs9364559 } \\
\hline GATG & $72.95(0.069)$ & $47.80(0.047)$ & 4.486 & 0.034 & 1.497 & $1.028-2.179$ \\
\hline \multicolumn{7}{|c|}{ rs3127596, rs9347438, rs9364559 } \\
\hline ATG & $176.52(0.166)$ & $138.94(0.133)$ & 4.384 & 0.036 & 1.293 & $1.016-1.645$ \\
\hline \multicolumn{7}{|c|}{ rs1367211, rs9347438, rs9364559 } \\
\hline GTG & $42.38(0.040)$ & $25.66(0.025)$ & 3.890 & 0.049 & 1.643 & $0.998-2.703$ \\
\hline \multicolumn{7}{|c|}{ rs6415085, rs9364559 } \\
\hline TG & $75.08(0.071)$ & $50.13(0.049)$ & 4.278 & 0.039 & 1.472 & $1.019-2.127$ \\
\hline
\end{tabular}

Frequency $<0.03$ in both control and casegroups has been dropped.

However, in haplotype association analysis, there is no difference for haplotype frequencies formed by five loci in the two groups. Less number of samples may be the main cause of the problem. Therefore, further research conducted in a lager sample size in different race is necessary.

In conclusion, our case-control study explored the association between five SNPs (rs1367211, rs3127596, rs9347438, rs6415085, and rs9364559) in the LPA gene and CAD in Chinese Han population for the first time. Rs9364559 in the LPA gene may be associated with risk of CAD in Chinese Han population, and with 4 haplotypes population formed by different blocks in the LPA gene may be associated with increased risk of CAD in Chinese Han population. Therefore, LPA gene is strongly associated with CAD in the Chinese Han population, which agrees with previous study.

\section{Conflict of Interests}

The authors declare that there is no conflict of interests regarding the publication of this paper.

\section{Authors' Contribution}

$\mathrm{Zi}$-Kai Song carried out the molecular genetic studies, participated in the sequence alignment, and drafted the paper. Hong-Yan Cao and Hai-Di Wu carried out the immunoassays and participated in the sequence alignment. Zi-Kai Song and Hai-Di Wu participated in the design of the study and performed the statistical analysis. Ling Qin conceived of the study and participated in its design and coordination and helped to draft the paper. All authors read and approved the paper.

\section{Acknowledgments}

The authors thank all participants for their supports and participation. Special thanks are due to the support from the Hepatology Department, Institute of Translational Medicine, the First Hospital, Jilin University, Changchun, China.

\section{References}

[1] M. Franchini, F. Peyvandi, and P. M. Mannucci, "The genetic basis of coronary artery disease: from candidate genes to whole genome analysis," Trends in Cardiovascular Medicine, vol. 18, no. 5, pp. 157-162, 2008.

[2] Q. Wang, "Molecular genetics of coronary artery disease," Current Opinion in Cardiology, vol. 20, no. 3, pp. 182-188, 2005.

[3] P. R. Burton, D. G. Clayton, L. R. Cardon et al., "Genome-wide association study of 14,000 cases of seven common diseases and 3,000 shared controls," Nature, vol. 447, no. 7145, pp. 661-678, 2007.

[4] R. McPherson, A. Pertsemlidis, N. Kavaslar et al., "A common allele on chromosome 9 associated with coronary heart disease," Science, vol. 316, no. 5830, pp. 1488-1491, 2007.

[5] A. Helgadottir, G. Thorleifsson, A. Manolescu et al., "A common variant on chromosome 9p21 affects the risk of myocardial infarction," Science, vol. 316, no. 5830, pp. 1491-1493, 2007.

[6] N. J. Samani, J. Erdmann, A. S. Hall et al., "Genomewide association analysis of coronary artery disease," New England Journal of Medicine, vol. 357, pp. 443-453, 2007.

[7] S. Erqou, S. Kaptoge, P. L. Perry et al., "Lipoprotein(a) concentration and the risk of coronary heart disease, stroke, and nonvascular mortality," Journal of the American Medical Association, vol. 302, no. 4, pp. 412-423, 2009.

[8] B. G. Nordestgaard, M. J. Chapman, K. Ray et al., "Lipoprotein(a) as a cardiovascular risk factor: current status," European Heart Journal, vol. 31, no. 23, pp. 2844-2853, 2010.

[9] R. Clarke, J. F. Peden, J. C. Hopewell et al., "Genetic variants associated with $\mathrm{Lp}(\mathrm{a})$ lipoprotein level and coronary disease," New England Journal of Medicine, vol. 361, no. 26, pp. 2518-2528, 2009.

[10] G. H. Dahlén and H. Stenlund, "Lp(a) lipoprotein is a major risk factor for cardiovascular disease: pathogenic mechanisms and clinical significance," Clinical Genetics, vol. 52, no. 5, pp. 272280, 1997.

[11] K. Berg, "A new serum type systerm in man the Lp systerm," Acta Pathologica, Microbiologica, et Immunologica Scandinavica, vol. 59, pp. 369-382, 1963.

[12] H. G. Kraft, H. J. Menzel, F. Hoppichler, W. Vogel, and G. Utermann, "Changes of genetic apolipoprotein phenotypes caused by liver transplantation. Implications for apolipoprotein synthesis," Journal of Clinical Investigation, vol. 83, no. 1, pp. 137142, 1989.

[13] H. H. Hobbs and A. L. White, "Lipoprotein(a): intrigues and insights," Current Opinion in Lipidology, vol. 10, no. 3, pp. 225236, 1999.

[14] E. Boerwinkle, C. C. Leffert, J. Lin, C. Lackner, G. Chiesa, and H. H. Hobbs, "Apolipoprotein(a) gene accounts for greater than $90 \%$ of the variation in plasma lipoprotein(a) concentrations," Journal of Clinical Investigation, vol. 90, no. 1, pp. 52-60, 1992. 
[15] D.-A. Trégouët, I. R. König, J. Erdmann et al., "Genome-wide haplotype association study identifies the SLC22A3-LPAL2LPA gene cluster as a risk locus for coronary artery disease," Nature Genetics, vol. 41, no. 3, pp. 283-285, 2009.

[16] M. M. Luke, J. P. Kane, D. M. Liu et al., "A polymorphism in the protease-like domain of apolipoprotein (a) is associated with severe coronary artery disease," Arteriosclerosis, Thrombosis, and Vascular Biology, vol. 27, no. 9, pp. 2030-2036, 2007.

[17] Y.-H. Chen, J.-M. Liu, R.-J. Hsu et al., "Angiotensin converting enzyme DD genotype is associated with acute coronary syndrome severity and sudden cardiac death in Taiwan: a casecontrol emergency room study," BMC Cardiovascular Disorders, vol. 12, article 6, 2012.

[18] L. Qi, J. Ma, Q. Qi, J. Hartiala, H. Allayee, and H. Campos, "Genetic risk score and risk of myocardial infarction in hispanics," Circulation, vol. 123, no. 4, pp. 374-380, 2011.

[19] J. Danesh, R. Collins, and R. Peto, "Lipoprotein(a) and coronary heart disease: meta-analysis of prospective studies," Circulation, vol. 102, no. 10, pp. 1082-1085, 2000.

[20] J. S. Danik, N. Rifai, J. E. Buring, and P. M. Ridker, "Lipoprotein(a), measured with an assay independent of apolipoprotein(a) isoform size, and risk of future cardiovascular events among initially healthy women," Journal of the American Medical Association, vol. 296, no. 11, pp. 1363-1370, 2006.

[21] P. M. Ridker, J. E. Buring, N. Rifai, and N. R. Cook, "Development and validation of improved algorithms for the assessment of global cardiovascular risk in women. The Reynolds Risk Score," Journal of the American Medical Association, vol. 297, no. 6, pp. 611-619, 2007.

[22] P. R. Kamstrup, M. Benn, A. Tybjærg-Hansen, and B. G. Nordestgaard, "Extreme lipoprotein(a) levels and risk of myocardial infarction in the general population. The Copenhagen City Heart Study," Circulation, vol. 117, no. 2, pp. 176-184, 2008.

[23] S. J. Nicholls, W. H. W. Tang, H. Scoffone et al., "Lipoprotein(a) levels and long-term cardiovascular risk in the contemporary era of statin therapy," Journal of Lipid Research, vol. 51, no. 10, pp. 3055-3061, 2010.

[24] J. Rubin, J. K. Han, T. A. Pearson, S. Holleran, R. Ramakrishnan, and L. Berglund, "Apo[a] size and PNR explain African American-Caucasian differences in allele-specific apo[a] levels for small but not large apo[a]," Journal of Lipid Research, vol. 47, no. 5, pp. 982-989, 2006.

[25] B. Enkhmaa, E. Anuurad, W. Zhang, T. Tran, and L. Berglund, "Lipoprotein(a): genotype-phenotype relationship and impact on atherogenic risk," Metabolic Syndrome and Related Disorders, vol. 9, no. 6, pp. 411-418, 2011.

[26] S. Lamon-Fava, S. M. Marcovina, J. J. Albers et al., "Lipoprotein(a) levels, apo(a) isoform size, and coronary heart disease risk in the Framingham Offspring Study," Journal of Lipid Research, vol. 52, no. 6, pp. 1181-1187, 2011.

[27] Z. G. Li, G. Li, and Y. L. Zhou, "Lack of association between lipoprotein(a) genetic variants and subsequent cardiovascular events in Chinese Han patients with coronary artery disease after percutaneous coronary intervention," Lipids in Health and Disease, vol. 12, article 127, 2013. 


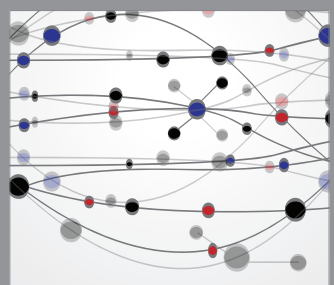

The Scientific World Journal
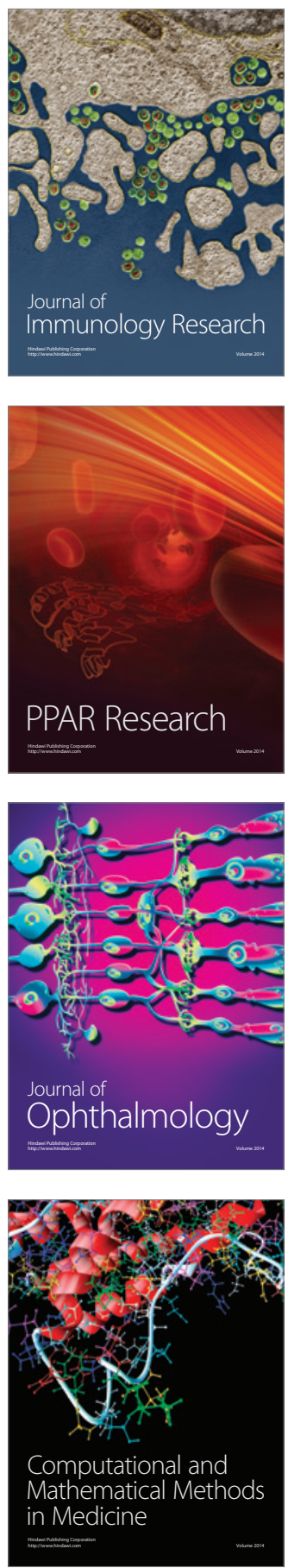

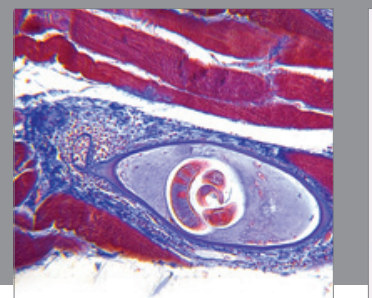

Gastroenterology

Research and Practice
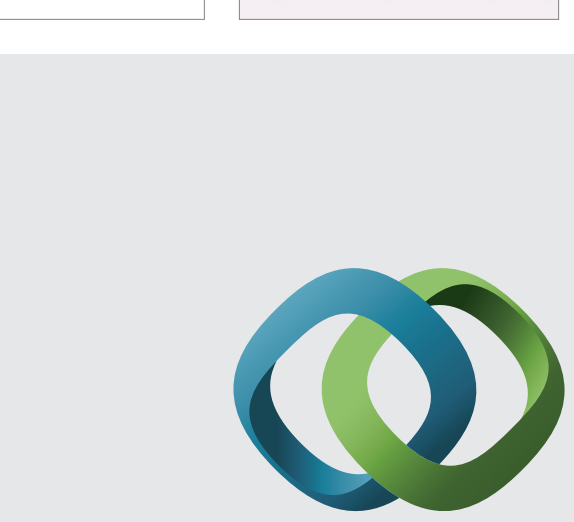

\section{Hindawi}

Submit your manuscripts at

http://www.hindawi.com
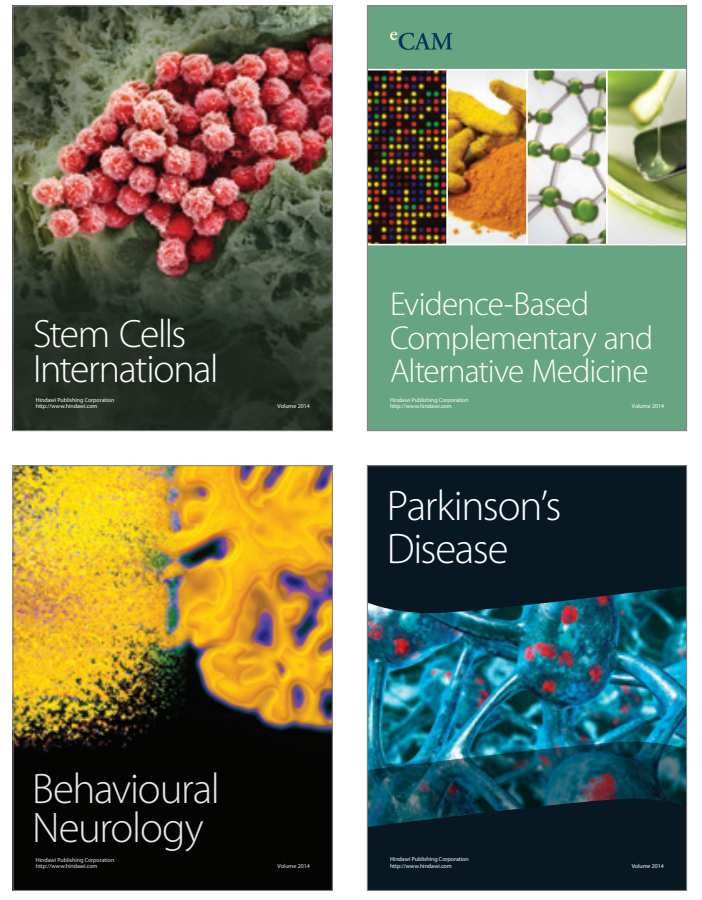
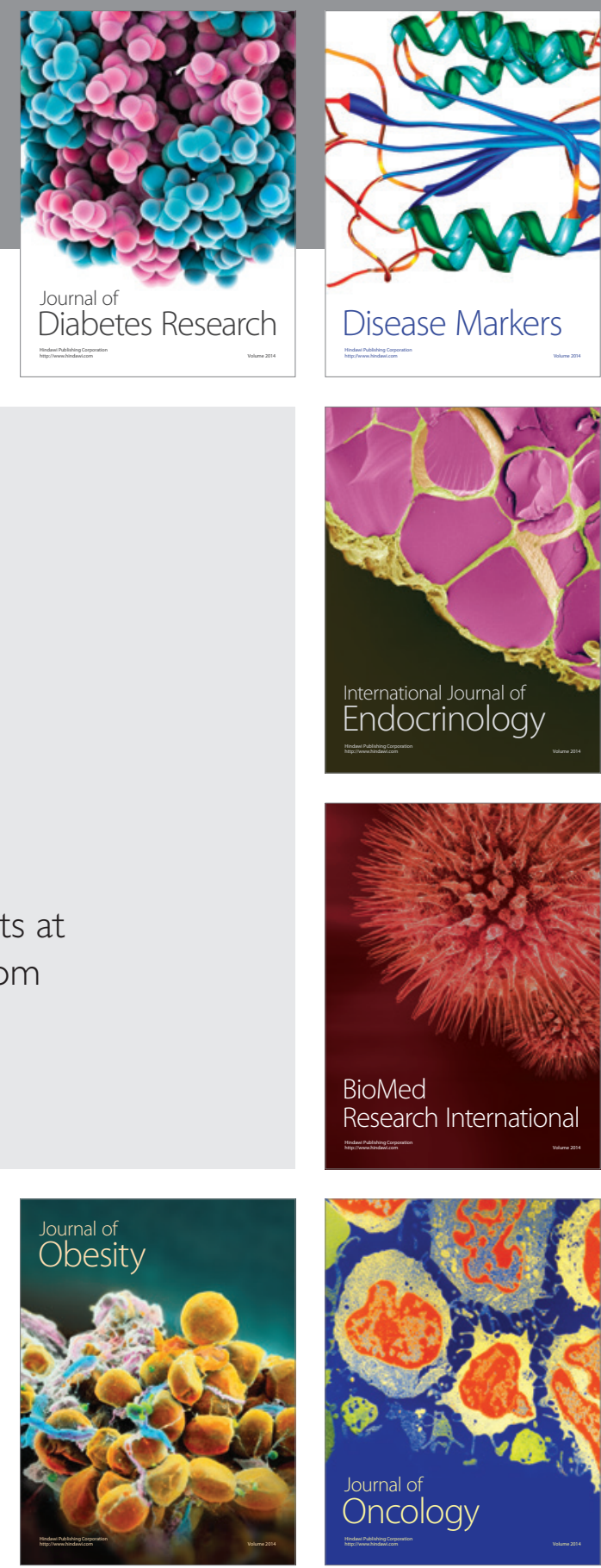

Disease Markers
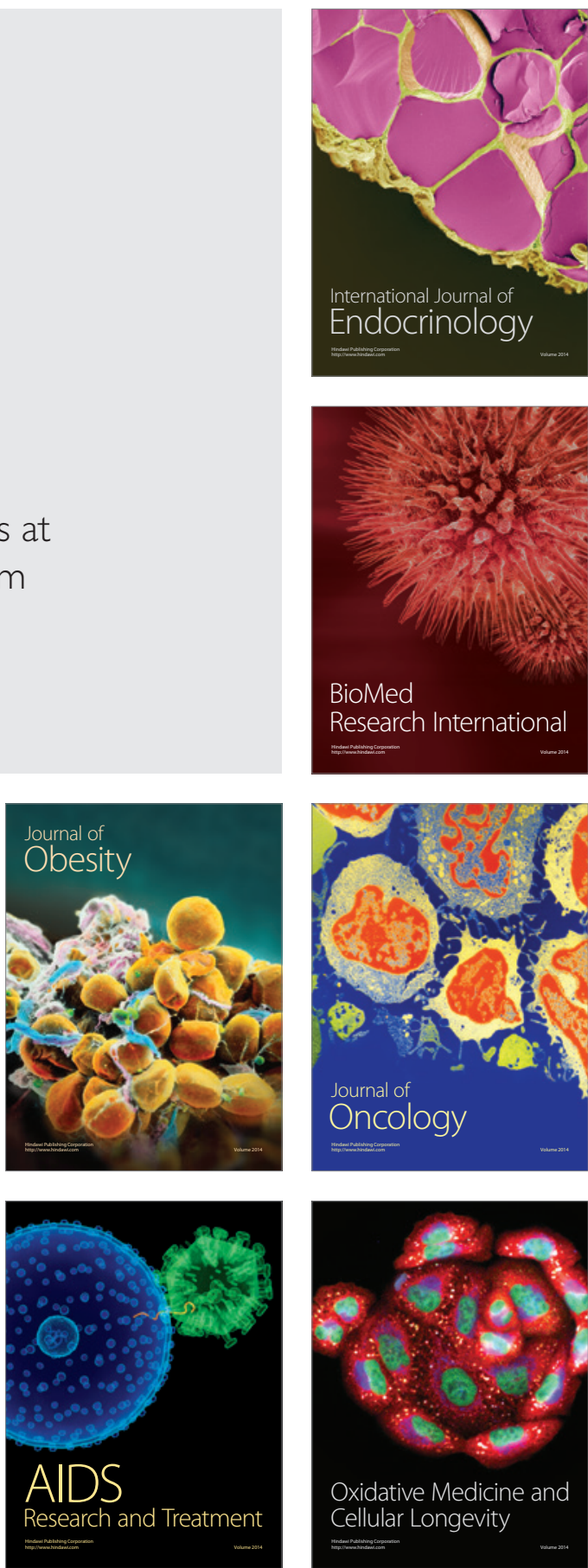